\title{
Evidence for the role of higher order reasoning processes in cue competition and other learning phenomena
}

\author{
JAN DE HOUWER \\ Ghent University, Ghent, Belgium \\ TOM BECKERS \\ University of Leuven, Leuven, Belgium \\ and \\ STEFAAN VANDORPE \\ Ghent University, Ghent, Belgium
}

\begin{abstract}
When people judge the contingency between a target cue and an outcome, they also take into account the contingency between the outcome and other cues with which the target cue co-occurred. Several authors have argued that such cue competition effects are due to higher order reasoning processes. We review the evidence that supports this hypothesis and discuss whether higher order reasoning might also play a role in other learning phenomena.
\end{abstract}

Imagine that you are a physician who is asked to determine which foods a certain patient is allergic to. You first see the results of several tests during which the patient ate mushrooms. On each of those tests, the patient showed an allergic reaction. Afterward, you receive information about several tests during which the patient ate both mushrooms and lettuce. Again, the patient always developed an allergic reaction. On other tests where the patient ate carrots, he did not develop an allergic reaction. To what extent would you think that the patient is allergic to lettuce?

This task is similar to the tasks that are used to examine cue competition in human contingency learning (HCL; see De Houwer \& Beckers, 2002b, and Dickinson, 2001, for reviews). In HCL studies, participants receive information about several situations in which certain cues (e.g., foods) and outcomes (e.g., an allergic reaction) can be present or absent. Afterward, participants are asked to answer certain questions about the relationship between the presence of cues and the presence of outcomes. As is common practice in the literature on HCL, we will use letters to refer to the presence of cues, a superscript "+" sign to refer to the presence of the outcome, and a superscript "-" sign to refer to the absence of an outcome. For instance, the expression "AT" " refers to a situation in which Cue A, Cue T, and the outcome are present, whereas " $\mathrm{A}^{-}$" signifies the presence of Cue A and the absence of the outcome.

\footnotetext{
We thank Marc Buehner and Tony Dickinson for their helpful comments on an earlier draft. Correspondence should be addressed to J. De Houwer, Department of Psychology, Ghent University, Henri Dunantlaan 2, B-9000 Ghent, Belgium (e-mail: jan.dehouwer@ugent.be).
}

Cue competition refers to the observation that judgments about the relationship between a target cue and an outcome depend not only on information about the presence or absence of the target cue and the outcome but also on information about the presence of other cues. The most well known cue competition effects are overshadowing and blocking. Overshadowing refers to the finding that contingency judgments for a target cue $\mathrm{T}$ are higher when $\mathrm{T}$ is presented on its own and followed by the outcome ( $\mathrm{T}^{+}$trials) than when $\mathrm{T}$ is always accompanied by an alternative cue $\mathrm{A}$ on those trials ( $\mathrm{AT}^{+}$trials). Blocking refers to the fact that the ratings for $\mathrm{Cue} \mathrm{T}_{\text {after }} \mathrm{AT}^{+}$ trials are nevertheless higher than after both $\mathrm{AT}^{+}$and $\mathrm{A}^{+}$ trials. For instance, in the example above, lettuce (Cue T) is always accompanied by mushrooms $\left(\mathrm{AT}^{+}\right)$and mushrooms were previously paired with the outcome $\left(\mathrm{A}^{+}\right)$. Studies have shown that, at least under certain conditions, participants will be less inclined to think that eating lettuce is related to allergic reactions if they have previously learned that eating mushrooms on its own leads to an allergic reaction (i.e., $\mathrm{A}^{+}$trials followed by $\mathrm{AT}^{+}$trials) than when they receive no information about tests on which only mushrooms are eaten (i.e., only $\mathrm{AT}^{+}$trials). This phenomenon is known as forward blocking (e.g., Dickinson, Shanks, \& Evenden, 1984). A similar result is obtained when the $\mathrm{AT}^{+}$trials precede the $\mathrm{A}^{+}$trials. Researchers have used the term backward blocking to refer to this effect (e.g., Shanks, 1985). Two other cue competition effects can occur when $\mathrm{AT}^{+}$trials are accompanied by $\mathrm{A}^{-}$trials (i.e., $\mathrm{A}$ is present but the outcome is absent). The term reduced overshadowing or prevention from overshadowing is used when $T$ receives a higher rating in a condition with $\mathrm{A}^{-}$trials followed by $\mathrm{AT}^{+}$trials than in 
a condition with only $\mathrm{AT}^{+}$trials. When the $\mathrm{AT}^{+}$trials precede the $\mathrm{A}^{-}$trials, this effect is called release from overshadowing or unovershadowing. There is, however, little research on reduced overshadowing and release from overshadowing (see Larkin, Aitken, \& Dickinson, 1998 , for an exception). We will thus focus on overshadowing and blocking effects.

\section{MODELS OF CUE COMPETITION EFFECTS}

\section{Associative Models}

Interest in cue competition effects in HCL started when associative models of animal conditioning were applied to HCL. Although there are several different associative models (see De Houwer \& Beckers, 2002b, and Pearce \& Bouton, 2001, for reviews), we will discuss only (variants of) the Rescorla-Wagner model (Rescorla \& Wagner, 1972). We chose this model because of its central role in modern learning research. When applied to HCL, the Rescorla-Wagner model implies that judgments about the relation between a cue and an outcome will reflect the strength of the association between the representations of those two stimuli. Importantly, changes in associative strength depend on the extent to which the outcome is expected on a certain trial. Therefore, when $\mathrm{AT}^{+}$trials are preceded by $\mathrm{A}^{+}$trials, the presence of the outcome is expected on the $\mathrm{AT}^{+}$trials because $\mathrm{A}$ has previously been established as a predictor of the outcome. As a result, $\mathrm{T}$ will be associated less with the outcome when $\mathrm{AT}^{+}$trials are preceded by $\mathrm{A}^{+}$trials than when there are no prior $\mathrm{A}^{+}$trials. The Rescorla-Wagner model thus provides a straightforward account for forward blocking.

A revised version of the model (Van Hamme \& Wasserman, 1994) can also explain backward blocking $\left(\mathrm{AT}^{+}\right.$ followed by $\mathrm{A}^{+}$). On the $\mathrm{AT}^{+}$trials, a within-compound association is formed between $\mathrm{A}$ and $\mathrm{T}$. Therefore, when $\mathrm{A}$ is presented on the subsequent $\mathrm{A}^{+}$trials, $\mathrm{T}$ is expected to occur but is actually absent. Van Hamme and Wasserman changed the Rescorla-Wagner model in such a way that the associative strength of cues that are expected but absent changes in the opposite direction of the associative strength of cues that are present. Hence, the associative strength of $\mathrm{T}$ decreases on $\mathrm{A}^{+}$trials that follow $\mathrm{AT}^{+}$trials and backward blocking occurs. On the basis of the revised model, one can predict that backward blocking depends on the strength of the within-compound association between A and T. Recent studies have confirmed that variables that affect this strength also influence backward blocking but not forward blocking (e.g., Aitken, Larkin, \& Dickinson, 2001; Dickinson \& Burke, 1996; Wasserman \& Berglan, 1998) and that backward but not forward blocking is related to measures of the strength of within-compound associations (e.g., Melchers, Lachnit, $\&$ Shanks, 2004). These results have been interpreted as strong evidence for the revised Rescorla-Wagner model (e.g., De Houwer \& Beckers, 2002b; Dickinson, 2001).

\section{Higher Order Reasoning Accounts}

In recent years, a number of nonassociative models of HCL have been proposed that provide an alternative account of cue competition effects in terms of higher order reasoning processes. We used the term higher order to refer to the fact that the processes can be characterized as reflective (e.g., Strack \& Deutsch, 2004), rule-based (e.g., Sloman, 1996), and deliberate (e.g., Smith \& DeCoster, 2000; also see Evans \& Over, 1996) and that they operate on conscious propositional knowledge in a controlled (i.e., slow, effortful, conscious, and/or intentional) manner. Higher order reasoning accounts are closely related to the well-known probabilistic contrast model of Cheng and colleagues (e.g., Cheng \& Novick, 1990). We will therefore first describe this model.

The central tenet of the probabilistic contrast model is that contingency judgments reflect the outcome of probabilistic contrasts, namely the difference between the probability of the outcome when the target cue is present $[P(\mathrm{O} \mid \mathrm{T})]$ and the probability of the outcome when the cue is absent $[P(\mathrm{O} \mid \sim \mathrm{T})]$ (e.g., Cheng, 1997; Cheng \& Novick, 1990). Importantly, when a target cue is always accompanied by another alternative cue, these probabilities will be calculated on the basis of a focal set of events in which the alternative cue is always present. That is, judgments about the relation between $\mathrm{T}$ and the outcome will reflect the difference between the probability of the outcome when both $\mathrm{A}$ and $\mathrm{T}$ are present $[P(\mathrm{O} \mid \mathrm{A} . \mathrm{T})]$ and the probability of the outcome when only A is present $[P(\mathrm{O} \mid \mathrm{A})]$. Both forward and backward blocking are attributed to the fact that these probabilities are equal when $\mathrm{AT}^{+}$trials are preceded or followed by $\mathrm{A}^{+}$trials, so that the probabilistic contrast equals 0 and the contingency judgment for $\mathrm{T}$ is low. When only $\mathrm{AT}^{+}$ trials are presented, an appropriate probabilistic contrast for T cannot be calculated. This will be reflected in uncertainty about the relation between $\mathrm{T}$ and the outcome (which is most often expressed as a contingency judgment close to the midpoint of the rating scale; e.g., Cheng, 1997).

The probabilistic contrast model is as such a normative model rather than a process model (e.g., Cheng, 1997; Cheng \& Holyoak, 1995). That is, it postulates that human contingency judgments should correspond to the outcome of appropriate probabilistic contrasts as calculated on the basis of the actual events. In its original form, however, it does not incorporate assumptions about how (i.e., by which processes) humans arrive at contingency judgments. It is not assumed, for instance, that participants actually calculate probabilities in a controlled manner. The fact that human contingency judgments often correspond to the outcome of appropriate probabilistic contrasts might as well be due to the operation of automatic associative learning processes (see Shanks, 1995, for such an argument). Some researchers, however, have extended the probabilistic contrast model by assuming that higher order reasoning processes do play an important role in HCL. 
Most prominently, Waldmann (2000; Waldmann \& Holyoak, 1992; see Waldmann \& Walker, 2005) has argued that contingency judgments depend not only on the objective information that is presented but also on how people interpret and reason about the presented information. How people interpret and reason about the events is a function of the causal model that they adopt. Causal models can be seen as a set of assumptions about the nature of the cues and outcomes and the relation between them. Sometimes participants will adopt a common-effect model in which the different cues are regarded as different potential causes of the same outcome. For instance, it is likely that participants adopt a common-effect model if instructions state that the cues are substances in the blood that can cause a disease (i.e., the outcome). However, people can also adopt a common-cause model, that is, interpret the cues as effects of a common cause. This is likely to happen when, for instance, participants are told that the cues are substances in the blood that could be the result of a particular disease (i.e., the outcome).

Waldmann (2000; Waldmann \& Holyoak, 1992; see Waldmann \& Walker, 2005) pointed out that cue competition will critically depend on the causal model that people adopt. When cues are seen as potential causes of the outcome and a target cue $\mathrm{T}$ is always presented on $\mathrm{AT}^{+}$trials, participants realize that the outcome on those trials could have been caused either by T or by A. Participants will thus be uncertain about the relation between $\mathrm{T}$ and the outcome. But a common-effect model also implies that the effect of different causes is most often additive. Therefore, participants realize that they might be able to infer the causal status of $\mathrm{T}$ by comparing the probability of the outcome in situations where both $\mathrm{A}$ and $\mathrm{T}$ are present with the probability of the outcome in situations where only A is present. Information about the alternative Cue $\mathrm{A}$ is thus taken into account when participants are making judgments about T. Hence, cue competition effects should be likely to occur when people adopt a common-effect model. When participants adopt a common-cause model, however, cue competition should be less likely. A single cause can have several independent effects. Hence, information about whether A is an effect of the outcome [i.e., $P(\mathrm{O} \mid \mathrm{A})$ ] is not relevant for evaluating whether $\mathrm{T}$ is an effect of the outcome. In recent papers, Waldmann (2000; Waldmann \& Walker, 2005 ) also explicitly put forward the idea that comparing probabilities involves controlled, effortful reasoning processes. Moreover, contingency judgments are thought to reflect not the objective contingency as determined by the actual events that have been presented, but the contingency as determined by what participants consciously believe happened (also see Cheng \& Holyoak, 1995, and De Houwer, 2002).

Lovibond and colleagues (Lovibond, 2003; Lovibond, Been, Mitchell, Bouton, \& Frohart, 2003; Mitchell \& Lovibond, 2002; also see De Houwer \& Beckers, 2003) proposed an inferential reasoning account of cue competition effects that shares many of its assumptions with causal model theory. Lovibond argued that "participants may combine the knowledge they have learned about individual cases to generate an inference about a particular cue in the same way as they solve other reasoning tasks" (2003, p. 98). In blocking studies, participants might for instance apply the following deductive rule (see De Houwer \& Beckers, 2003, p. 346):

If Cue $\mathrm{A}$ on its own causes the outcome to occur with a certain intensity and probability, and if Cue A and T together cause the outcome to occur with the same intensity and probability, this implies that Cue $\mathrm{T}$ is not a cause of the outcome.

Likewise, in reduced overshadowing and release from overshadowing studies, the following rule might be applied:

If Cue A on its own does not cause or predict the outcome but Cue $\mathrm{A}$ and $\mathrm{T}$ together do cause or predict the outcome, this implies that Cue $\mathrm{T}$ is a cause or predictor of the outcome.

These deductive rules are highly similar to the probabilistic contrasts that form the core of the probabilistic contrast model and causal model theory. In fact, when restricted to probabilities, the rules are a nonstatistical formulation of the principle that contingency judgments in cue competition situations depend on a comparison of probabilities [i.e., the probability of the outcome when only A is present, $P(\mathrm{O} \mid \mathrm{A})$, and when both $\mathrm{A}$ and $\mathrm{T}$ are present, $P(\mathrm{O} \mid \mathrm{A} . \mathrm{T})]$. Like causal model theory, the inferential reasoning account postulates that participants actually compare (estimates of) these probabilities in an controlled and effortful manner and that they are aware of the conditions under which these inferences are likely to generate valid results.

Researchers such as Waldmann (2000) and Lovibond (2003) have thus explicitly put forward the hypothesis that higher order reasoning processes are responsible for cue competition effects in HCL. Because of their unique emphasis on reasoning, these models have led to many new predictions. In the following section, we will discuss these predictions and the evidence that relates to them.

\section{EVIDENCE FOR THE ROLE OF HIGHER ORDER REASONING IN CUE COMPETITION EFFECTS}

First, we will focus on the assumption that participants are aware of the conditions under which certain inferences are likely to be valid. Second, we will deal with the role of the conscious propositional knowledge about cue-outcome relations. Third, we will discuss evidence related to the controlled nature of higher order reasoning.

\section{Knowledge About the Conditions Under Which Inferences Are Valid}

Causal nature of the cues. As we pointed out above, a central prediction of the causal model theory is that cue competition effects depend on the causal model that par- 
ticipants adopt. When participants interpret the cues as potential causes of the outcome (e.g., different substances in the blood that are potential causes of a disease), they should be uncertain about the causal status of $\mathrm{T}$ when $\mathrm{T}$ occurs only on $\mathrm{AT}^{+}$trials (unless they can verify that the outcome is more probable or intense on the $\mathrm{AT}^{+}$trials than on $\mathrm{A}^{+}$trials; see below). But when the cues are regarded as potential effects of the outcome (e.g., different substances in the blood that can be caused by a disease), $\mathrm{AT}^{+}$trials suffice to infer that $\mathrm{T}$ is an effect of the outcome. Waldmann (Waldmann, 2000; Waldmann \& Holyoak, 1992; see De Houwer, Beckers, \& Glautier, 2002, for related results) indeed found cue competition (i.e., lower ratings for $\mathrm{T}$ than for $\mathrm{A}$ after $\mathrm{AT}^{+}$and $\mathrm{A}^{+}$trials) ${ }^{1}$ only when the cues were described as causes of the outcome but not when they were said to be effects of the outcome. Although some researchers have found cue competition when cues were described as effects of the outcome (e.g., Cobos, López, Caño, Almaraz, \& Shanks, 2002; Shanks \& López, 1996; Tangen \& Allan, 2004), one could argue that cue competition emerged because participants chose not to adopt the causal framework that was suggested by the instructions (either because the instructions were not clear or because elements of the design were incompatible with the suggested causal framework, see Waldmann $\&$ Walker, 2005). At the very least, one can conclude that the causal status of the cues can under certain conditions have an impact on contingency judgments (e.g., Tangen $\&$ Allan, 2004).

Note that from a reasoning point of view, the causal status of the cues should be less important in reduced overshadowing (i.e., $\mathrm{AT}^{+}$trials are preceded by $\mathrm{A}^{-}$trials). When cues are regarded as effects of the outcome (common-cause model), $T$ must be related to the outcome because it always occurs when the outcome occurs (i.e., on the $\mathrm{AT}^{+}$trials). When cues are regarded as potential causes of the outcome (common-effect model), one can infer on the basis of the $\mathrm{A}^{-}$trials that $\mathrm{A}$ is not a cause of the outcome. Hence, $T$ must be the cause of the outcome on the $\mathrm{AT}^{+}$trials (assuming that the compound of $\mathrm{A}$ and $\mathrm{T}$ is not regarded as a different, unique cue that is more than the sum of its elements; see Williams, Sagness, \& McPhee, 1994). Therefore, reduced overshadowing (i.e., higher ratings for $\mathrm{T}$ after $\mathrm{AT}^{+}$and $\mathrm{A}^{-}$trials than after only $\mathrm{AT}^{+}$trials) should occur irrespective of the causal model that participants adopt. De Houwer et al. (2002, Experiment 3) indeed found that the causal nature of the cues (cause or predictor of the outcome) did not have an effect on reduced overshadowing even though it did have an effect on blocking.

Ceiling effects. According to a higher order reasoning account of blocking, participants infer that $\mathrm{T}$ is not a cause of the outcome because the probability or intensity of the outcome is the same when both $\mathrm{A}$ and $\mathrm{T}$ are present than when only A is present. Participants can make this inference because the effect of different causes tends to be additive. However, even if one makes the assumption that cues are causes whose effect is additive, it is possible that one cannot observe the added effect of $\mathrm{T}$ because A on its own already always causes the outcome to the maximal extent. That is, ceiling effects can prevent one from making a valid inference about the causal status of T. Several studies show that participants are aware of this limitation and take it into account when making contingency judgments.

Wu and Cheng (1999) examined the impact of ceiling considerations by describing to their participants certain scenarios in which a researcher wanted to test the extent to which a certain drug would cause something to happen (e.g., increase the fertility of a certain breed of cats; generative causes) or the extent to which a drug could prevent something from happening (e.g., prevents cats from becoming pregnant; preventative causes). The test involved two conditions, a treatment condition in which the drug was administrated and a control condition in which the drug was not administrated. The participant received feedback about the probability of the outcome (e.g., the percentage of cats that became pregnant) in each of the two conditions. In these scenarios, the drug functioned as the target cue $\mathrm{T}$ whereas the alternative cue A was the context common to both conditions (e.g., the breed of cats). The treatment condition can thus be regarded as consisting of AT trials and the control condition as consisting of A trials. In some scenarios, the outcome occurred in every single case in both the experimental and control conditions. When this was the case, participants indicated that the researcher could not infer with any certainty that the drug had a generative effect (e.g., increased the fertility of cats) because the outcome was already at maximal levels in the control condition. But participants were confident that such a result does allow one to conclude that the drug does not have a preventative effect (e.g., prevents the cats from becoming pregnant) because there was clearly room for a decrease in the likelihood of the outcome in the treatment condition. The opposite result was found when the outcome never occurred in either condition. In such cases, participants judged that the researcher could make valid conclusions about generative causes but not about preventative causes. These results show that participants take into account ceiling considerations, not only for generative causes but also for preventative causes.

De Houwer et al. (2002) examined this issue by manipulating the intensity rather than the probability of the outcome. Moreover, they used a tank paradigm in which information was presented on a trial-by-trial basis. All participants saw several trials on which Weapon A destroyed the tank with an impact of 10 . They also saw trials on which Weapons $\mathrm{A}$ and $\mathrm{T}$ fired together and also destroyed the tank with an impact of 10. Finally, they saw trials on which Weapon $Z$ did not destroy the tank (impact of 0 ) and trials on which Weapons $\mathrm{K}$ and $\mathrm{L}$ together destroyed the tank (impact of 10). In one condition, participants were told that the maximal impact that could be measured corresponded to a value of 10 . Because Weapon A on its own already caused the outcome to the 
maximal extent, it was not possible to make a definite conclusion about the relation between $\mathrm{T}$ and the outcome. Hence, blocking (i.e., lower ratings for $\mathrm{T}$ than for $\mathrm{K}$ and $\mathrm{L}$ ) should not occur. In the second condition, the maximal impact that could be measured corresponded to a value of 20 . Because both A on its own and A and T together caused the outcome to a submaximal extent, participants could infer that $\mathrm{T}$ did not have an effect on the outcome and blocking should thus occur. The results confirmed these predictions: Blocking was much stronger and significant only when outcomes occurred to a submaximal extent. Moreover, participants were more confident in the accuracy of their rating for Cue $\mathrm{T}$ than for Cues $\mathrm{K}$ and $\mathrm{L}$, but only in the submaximal condition. This is in line with the idea that participants realized that the inference on which they based their contingency rating for $\mathrm{T}$ (i.e., $\mathrm{T}$ is not a cause of the outcome because one can verify that it does not add anything to the effect of A) was likely to be valid in the submaximal (but not maximal) condition.

One might argue that associative models can account for the effect of the maximality of the outcome if one assumes that submaximal outcomes are encoded as being less intense than the maximal outcome (and thus as different in associability or the maximal level of associative strength that can be achieved). Without going into details, it is true that this additional assumption allows the Rescorla-Wagner model (Rescorla \& Wagner, 1972) to explain the fact that ratings for $\mathrm{T}$ are higher with maximal (i.e., intense) outcomes than with submaximal (i.e., less intense) outcomes. But simulations show that the Rescorla-Wagner model predicts an even stronger increase in the ratings for the control cues $\mathrm{K}$ and $\mathrm{L}$, such that blocking (i.e., rating for $\mathrm{T}$ compared with ratings for $\mathrm{K}$ and $\mathrm{L}$ ) is reduced when the intensity of the outcome increases. This prediction is clearly inconsistent with the data.

One might also argue that the absence of a blocking effect in the study of De Houwer et al. (2002) is at odds with the fact many other studies have demonstrated significant blocking effects. A first point to note, however, is that this does not call into doubt the crucial finding that blocking depends on maximality of the outcome. A second point is that the evidence for blocking with maximal outcomes is quite limited. In virtually all published studies on blocking, the ratings for a blocked cue $\mathrm{T}\left(\mathrm{A}^{+}\right.$, $\mathrm{AT}^{+}$) were compared with the ratings of a cue $\mathrm{Y}$ that was involved in a reduced overshadowing or release from overshadowing training $\left(\mathrm{B}^{-}, \mathrm{BY}^{+}\right)$. The results of these studies consistently show that ratings for $\mathrm{Cue} T$ are lower than ratings for Cue Y (e.g., Williams et al., 1994). But this effect could be due either to blocking (i.e., ratings for $\mathrm{T}$ are lower because of the $\mathrm{A}^{+}$trials) or to reduced overshadowing or release from overshadowing (i.e., ratings for $\mathrm{Y}$ are higher because of the $\mathrm{B}^{-}$trials). On the basis of higher order reasoning accounts, one would expect that, in these studies, cue competition was due in most part to reduced overshadowing or release from overshadowing rather than to blocking. It is unlikely that blocking occurred in these studies because the outcome always occurred with a maximal intensity on both the $\mathrm{A}^{+}$ and $\mathrm{AT}^{+}$trials. Ceiling considerations do not apply to reduced overshadowing or release from overshadowing. The $\mathrm{B}^{-}$trials allow one to infer that $\mathrm{B}$ is not a cause of the outcome. Hence, one can infer that $\mathrm{Y}$ is a cause of the outcome even though the outcome is maximal on the $\mathrm{BY}^{+}$trials. Studies that included the appropriate controls to determine the contribution of blocking and release from overshadowing separately indeed seem to confirm this analysis (e.g., Larkin et al., 1998; but see Wasserman \& Berglan, 1998).

Additivity training. Even when ceiling considerations do not apply, participants might have doubts about whether the effect of different causes is additive. Lovibond et al. (2003) explicitly manipulated beliefs about the additivity of causes by exposing participants to a training phase during which the effect of different causes was shown to be additive or not additive. All participants first received information about a patient who developed an allergic reaction of normal intensity after eating Food I $\left(\mathrm{I}^{+}\right)$and after eating Food $\mathrm{J}\left(\mathrm{J}^{+}\right)$. The participants in the additive group then learned that the patient showed a stronger allergic reaction after eating both Foods I and J $\left(\mathrm{IJ}^{++}\right)$. In the nonadditive group, participants learned that an allergic reaction of normal magnitude occurred after eating Foods I and $\left.\mathrm{J}_{(\mathrm{IJ}}{ }^{+}\right)$. During the next phase, all participants saw trials on which Food A caused an allergic reaction $\left(\mathrm{A}^{+}\right)$, trials on which Foods $\mathrm{A}$ and $\mathrm{T}$ together caused an allergic reaction of the same magnitude $\left(\mathrm{AT}^{+}\right)$, and trials on which Foods $\mathrm{C}$ and $\mathrm{D}$ together caused an allergic reaction of a normal magnitude $\left(\mathrm{CD}^{+}\right)$. Blocking was measured by comparing the causal rating for Cue $\mathrm{T}$ with the causal ratings of Cues $\mathrm{C}$ and $\mathrm{D}$. Results showed that participants in the additive group showed a significantly stronger blocking effect than participants in the nonadditive group.

\section{On the Role of Conscious Propositional Knowledge}

Higher order reasoning occurs on the basis of conscious propositional knowledge. Therefore, if cue competition depends on higher order reasoning, it should be influenced by the conscious propositional knowledge that participants hold. Several studies support this hypothesis.

Assumptions about the presence or absence of cues. De Houwer (2002) conducted a forward blocking study with submaximal outcomes in which participants could not determine whether Cue $\mathrm{T}$ was present or absent during the $\mathrm{A}^{+}$trials that preceded the $\mathrm{AT}^{+}$trials. At the end of the experiment, participants were asked whether they believed that Cue $\mathrm{T}$ was present during the $\mathrm{A}^{+}$trials. Only those participants who believed that $\mathrm{T}$ was absent showed a blocking effect. In a second experiment, participants received verbal information about the presence of $\mathrm{T}$ during $\mathrm{A}^{+}$trials. Despite the fact that they received this information only after all $\mathrm{A}^{+}$and $\mathrm{AT}^{+}$trials had 
been presented, participants who were told that $\mathrm{T}$ was absent on the $\mathrm{A}^{+}$trials showed a strong blocking effect, whereas no blocking was found in a group of participants who were told that $\mathrm{T}$ was present during the $\mathrm{A}^{+}$trials. These findings are in line with a higher order reasoning account. In order to make an inference about the causal status of Cue T, participants need to have conscious propositional knowledge about the likelihood and intensity of the outcome when A and T are present and when only A is present. Participants who believe that $\mathrm{T}$ was present on the $\mathrm{A}^{+}$trials do not have conscious propositional knowledge about what happens when only $\mathrm{A}$ is present can therefore not make a valid inference and will thus not show blocking.

In a related experiment, Waldmann (2000, Experiment 2) described cues as potential effects of a common cause and asked participants to judge the extent to which a cue allowed one to predict that the cause was present. Prior research had shown that after $\mathrm{AT}^{+}$and $\mathrm{A}^{+}$trials, contingency ratings were as high for $\mathrm{Cue} \mathrm{T}$ as for Cue $\mathrm{A}$ (i.e., no cue competition). This was predicted on the basis of causal model theory because information about whether A is an effect of the outcome should have no bearing on whether $\mathrm{T}$ is also an effect of the same outcome. Waldmann pointed out, however, that participants do need to believe that $\mathrm{T}$ was actually present on the $\mathrm{A}^{+}$ trials. If they believe that $\mathrm{T}$ was absent on the $\mathrm{A}^{+}$trials, they should infer that the outcome causes $\mathrm{T}$ on some trials (i.e., $\mathrm{AT}^{+}$trials) but not other trials (i.e., $\mathrm{A}^{+}$trials), whereas the outcome does always cause $\mathrm{A}$ (i.e., both $\mathrm{A}^{+}$ and $\mathrm{AT}^{+}$trials). Hence, they should judge that $\mathrm{A}$ is a more reliable indicator of the outcome than T. To examine this issue, Waldmann (Experiment 2) created one condition in which participants were explicitly informed that $\mathrm{T}$ was absent on the $\mathrm{A}^{+}$trials (explicit-absence condition) and a second condition in which participants were told that no information was available about the presence of $\mathrm{T}$ on the $\mathrm{A}^{+}$trials (no-information condition). Whereas cue competition (i.e., lower ratings for $\mathrm{T}$ than for $\mathrm{A}$ after $\mathrm{A}^{+}$and $\mathrm{AT}^{+}$trials) did emerge in the explicitabsence condition, it was not found in the no-information condition.

Recursive higher order reasoning. When participants are motivated and have the opportunity to reason, they will use any available means to arrive at conscious propositional knowledge that can serve as the input for the application of a valid deductive rule. One of these means is prior higher order reasoning. This idea is illustrated by studies of De Houwer and Beckers (2002a, 2002c), who described a phenomenon called higher order retrospective revaluation. In their experiments, participants saw $\mathrm{AB}^{+}, \mathrm{AT}^{+}$, and $\mathrm{B}^{-}$or $\mathrm{B}^{+}$trials. The results showed that the nature of the trials with $\mathrm{Cue} B\left(\mathrm{~B}^{+}\right.$or $\mathrm{B}^{-}$) affected not only causal ratings for $\mathrm{A}$ but also the ratings for $T$. The direction of the effect on the ratings for $\mathrm{T}$ (higher ratings after $\mathrm{B}^{+}$trials than after $\mathrm{B}^{-}$trials) was opposite the effect on the ratings for A (lower ratings after $\mathrm{B}^{+}$trials than after $\mathrm{B}^{-}$trials). At first sight, partic- ipants cannot arrive at a valid conclusion about the causal status of $\mathrm{T}$ because no $\mathrm{A}^{+}$trials are presented. When, however, participants see $\mathrm{AB}^{+}$and $\mathrm{B}^{-}$trials, they can infer that $\mathrm{A}$ is a cause of the outcome because the outcome occurs on the $\mathrm{AB}^{+}$trials despite the fact that $\mathrm{B}$ on its own does not cause the outcome. Given this inferred propositional knowledge, they can subsequently infer that $\mathrm{T}$ is not a cause of the outcome because the observed effect of $\mathrm{A}$ and $\mathrm{T}$ together is the same as the hypothesized effect of $\mathrm{A}$ alone. If $\mathrm{B}^{+}$trials are presented rather than $\mathrm{B}^{-}$trials, participants can conclude that $\mathrm{A}$ is not a cause of the outcome (because B alone has the same effect as $\mathrm{A}$ and $\mathrm{B}$ together) and thus that $\mathrm{T}$ is a cause of the outcome (because the outcome is more likely when A and $\mathrm{T}$ together are presented than when $\mathrm{A}$ is presented on its own). Note that Macho and Burkart (2002), who also obtained evidence for higher order retrospective revaluation, developed a formalized probabilistic model of the idea that inferences can serve as the input for further inferences.

Retrospective revaluation depends on memory for compounds. When we described the revised version of the Rescorla-Wagner model (Van Hamme \& Wasserman, 1994), we noted that this model is supported by the fact that backward blocking and release from overshadowing depend on the strength of within-compound associations (e.g., Dickinson \& Burke, 1996; Melchers et al., 2004). The strength of a within-compound association is, however, measured primarily by assessing whether participants can remember which cue went together with which other cue. Hence, one could also say that studies on the role of within-compound associations in cue competition demonstrate that backward blocking and release from overshadowing (but not forward blocking and reduced overshadowing) depend on the memory for the compounds. This is exactly what one would predict on the basis of accounts that attribute cue competition to higher order reasoning. In order to make a valid inference about the status of Cue $\mathrm{T}$, one needs to have the propositional knowledge that $\mathrm{A}$ and $\mathrm{T}$ together are followed by the outcome and that $\mathrm{A}$ on its own does or does not lead to the outcome. In a backward blocking or release from overshadowing design, one can possess the necessary knowledge only once the $\mathrm{A}^{-}$or $\mathrm{A}^{+}$trials are experienced. Because these trials come after the $\mathrm{AT}^{+}$trials, during the $\mathrm{A}^{-}$or $\mathrm{A}^{+}$trials, one needs to remember that $\mathrm{A}$ and $\mathrm{T}$ went together and were followed by the outcome. If one does not remember the $\mathrm{AT}^{+}$trials, a valid inference cannot be made. Hence, backward blocking and release from overshadowing should depend on memory for compounds. Forward blocking and reduced overshadowing should not depend on the memory for compounds because participants can make a valid inference at the moment when the $\mathrm{AT}^{+}$trials are presented. They only need to remember the $\mathrm{A}^{+}$trials. Also note that remembering a compound is probably more difficult than remembering a trial on which only one cue was presented. This could explain why backward blocking is 
often smaller in magnitude than forward blocking and why release from overshadowing is often smaller than reduced overshadowing. Such a difference is typically found in studies in which many cues are used (and memory for compounds might indeed have been worse than memory for elements; e.g., Dickinson \& Burke, 1996) but is often not found in studies with only a few cues (and both memory for compounds and elements might be good; e.g., De Houwer et al., 2002).

\section{Support for the Controlled Nature of Higher Order Reasoning}

The core characteristic of higher order reasoning is that it is a controlled (in the sense of effortful, conscious, slow, intentional, and controllable) process. Assuming that cue competition is due to higher order reasoning, one can thus predict that cue competition should be less likely to occur when participants are not motivated or do not have the necessary cognitive resources to reason. De Houwer and Beckers (2003) examined whether blocking depends on cognitive resources. They asked participants to perform a difficult (i.e., speeded responding to the pitch of tones presented at random intervals) or easy (i.e., speeded responding to the presence of tones presented at fixed intervals) secondary task while being exposed to $\mathrm{A}^{+}, \mathrm{AT}^{+}, \mathrm{KL}^{+}$, and $\mathrm{Z}^{-}$trials and while making judgments about the causal status of the cues. In line with the predictions, they found more blocking (i.e., lower ratings for $\mathrm{T}$ than for $\mathrm{K}$ and $\mathrm{L}$ ) when participants were exposed to the easy secondary task than when the secondary task was difficult.

The fact that higher order reasoning is a controlled process also allows one to predict that participants should in principle be able to report on this process. Moreover, variables that influence reasoning should also influence the verbal reports. Also, because controlled processes are time-consuming, one can predict that the time that participants need to make a contingency judgment will depend on the complexity of the reasoning processes that are assumed to be necessary to arrive at an accurate judgment. It would be interesting to test these predictions in future research.

\section{SCOPE OF THE HIGHER ORDER REASONING ACCOUNT}

In our opinion, the findings that were summarized above demonstrate that higher order reasoning is a source of cue competition effects in HCL. This conclusion is important because it considerably increases our knowledge about the processes that underlie HCL. The question remains, however, as to how important reasoning is in HCL and associative learning in general. ${ }^{2}$ In this section, we will discuss whether there are processes other than higher order reasoning that can produce cue competition effects and whether reasoning could play a role in phenomena other than cue competition in HCL. Finally, we will speculate about the processes that might underlie higher order reasoning itself and discuss the limitations of existing higher order reasoning accounts.

\section{Other Sources of Cue Competition in HCL}

Although we believe that cue competition effects are often due to higher order reasoning, we do not want to claim that this is the only source of such effects. For instance, there is evidence that blocking in HCL can be due to attentional processes (e.g., Glautier, 2002; Kruschke \& Blair, 2000; Le Pelley \& McLaren, 2003). Such attentional processes could, however, be integrated in higher order reasoning accounts of HCL. From a reasoning perspective, participants can form conscious propositional knowledge about cue-outcome relations on the basis of observed events in which those stimuli are present or absent. This knowledge can then provide the input for further higher order reasoning. Conscious propositional knowledge can, however, also determine the selection of the events to which one directs attention. For instance, if participants entertain the conscious hypothesis that $\mathrm{A}$ is a valid predictor of the outcome and are asked to make predictions about the presence of the outcome, they might intentionally direct their attention to A. This could in some cases result in a failure to register that $\mathrm{Cue} T$ was present on the $\mathrm{AT}^{+}$trials. In such cases, participants will be unable to form propositional knowledge about Cue $\mathrm{T}$. Hence, $\mathrm{T}$ will be treated as a new cue, which could result in blocking. Note that this attentional account is different from associative attentional models of learning (e.g., Kruschke, 2001; Mackintosh, 1975). For instance, whereas associative attentional models of learning emphasize the role of automatic (in the sense of effortless, unconscious, unintentional, or uncontrollable) processes, an inferential account implies that the impact of attentional processes on contingency judgments will be mediated by controlled processes (i.e., conscious propositional knowledge).

As we noted earlier, cue competition effects in HCL have traditionally been interpreted as evidence for acquisition-focused associative models such as the Rescorla-Wagner (1972) model that postulate that cues compete for the acquisition of associative strength (see De Houwer \& Beckers, 2002b, for a review). These models are built on the idea that the learning of cue-outcome relations (i.e., changes in the strength of the association between the representations of the cue and the outcome) is a direct function of the extent to which the presence (or absence) of the outcome was anticipated. Forward blocking, for example, is attributed to the fact that participants do not learn that $\mathrm{T}$ is associated with the outcome because the presence of the outcome on those trials was fully anticipated on the basis of Cue A. In essence, this account implies that humans (and other animals) are incapable of registering the contingency between the presence of $\mathrm{T}$ and the presence of outcome whenever the outcome is already expected. Although this idea has great historical value and has proven to be in line with many empirical findings, it seems implausible. On the one 
hand, one could argue that organisms should not waste valuable resources on learning redundant information that is not immediately useful. But on the other hand, redundant information could be essential in the long run (e.g., when information about the original cue is no longer available). Therefore, in situations where people do have the resources to learn redundant information (e.g., when they are motivated to learn or other demands on resources are sparse), we see no reason why they should not be able to learn this information. It is because associative models such as the Rescorla-Wagner model basically deny people the ability to learn redundant cue-outcome relations that we have strong reservations about whether these models provide an accurate account of cue competition effects (see Miller \& Matzel, 1988, and Denniston, Savastano, \& Miller, 2001, for a type of associative model that does not deny people this ability). According to higher order reasoning accounts, cue competition effects are not due to a failure to learn (provided that enough attention is allocated to the presented information; see above), but to the combination of different pieces of conscious propositional knowledge during reasoning.

Although there thus are a priori reasons to argue that competitive associative learning of the kind proposed in the Rescorla-Wagner model does not provide an accurate account of cue competition, it is difficult to exclude the possibility that it does sometimes form the basis of cue competition effects. Some researchers have pointed out that cue competition effects can be found even in situations where higher order reasoning is unlikely to take place. For instance, Le Pelley and McLaren (2003; also see Dickinson \& Burke, 1996; Larkin et al., 1998; Wasserman \& Berglan, 1998) found significant cue competition in a study in which 16 different cues were used and 16 different compound stimuli were presented. They argued that the large number of cues "helped to ensure a large memory load, hopefully preventing subjects from basing their ratings on inferences made from explicit episodic memories ... Instead subjects should have to rely on associative processes to provide a more 'automatic' measure of causal efficacy for each cue" (Le Pelley \& McLaren, 2003, p. 74; also see Dickinson, 2001, p. 23). One should note, however, that Le Pelley and McLaren did not provide independent evidence to support the assumption that higher order reasoning did not occur in their experiments. It thus remains to be seen whether evidence for the role of higher order reasoning (e.g., an impact of ceiling effects) can be found also in studies with many cues.

To conclude, we believe that there is strong evidence to support the hypothesis that cue competition effects are due either to the fact that participants make an inference about a cue-outcome relation on the basis of available conscious propositional knowledge or to the fact that they do not pay attention to cues that are always presented in compound with other cues that have previously been established as causes or predictors of the outcome. At present, we do not know of any evidence that uniquely supports the hypothesis that cue competition can be due to competitive associative learning. Although we do not exclude the possibility that such evidence will emerge in the future, studies directed at obtaining such evidence should include controls that allow one to exclude the possibility that the observed effects are due to higher order reasoning or attentional processes.

\section{On the Role of Higher Order Reasoning in Other Forms of Associative Learning}

Other phenomena in HCL. Until now, we have focused on cue competition only. Although this is a theoretically important phenomenon, there are of course many other phenomena that have been demonstrated in HCL. Because a detailed discussion of all these phenomena would fall outside the scope of this paper, we will only briefly discuss how one could account for acquisition and trial-order effects on the basis of higher order reasoning.

So far we have simply assumed that participants can form conscious propositional knowledge on the basis of (among other things) observation of cue-outcome events, but we have not dealt with how this acquisition of knowledge can be achieved. One possibility is that conscious knowledge about observed cue-outcome relations is the result of the operation of simple associative processes. Although we do not rule out this possibility, there is an alternative, not mutually exclusive, explanation in terms of higher order reasoning. In order to determine whether there is an association between a Cue A and an outcome $\mathrm{O}$, people might compare the probability that $\mathrm{O}$ is present when $\mathrm{A}$ is also present $[P(\mathrm{O} \mid \mathrm{A})]$ with the probability that $\mathrm{O}$ is present when $\mathrm{A}$ is absent $[P(\mathrm{O} \mid \sim \mathrm{A})]$. This hypothesis corresponds to the probabilistic $\Delta P$ model except that it does not stipulate what people should do (i.e., is not merely a normative hypothesis) but rather what people actually do (i.e., it specifies the nature of the actual processes). In fact, it can be regarded as a form of higher order reasoning that depends on the following deductive rule: "If the probability of $B$ is higher when $A$ is present than when A is absent, then A and B are associated."

In order to estimate the relevant probabilities, one needs to access memories of past situations in which the events were present or absent. This access can be either controlled or automatic. Controlled retrieval of memory traces would imply that one intentionally and consciously tries to recollect relevant past events. Once recalled, one can combine the relevant information in a controlled manner and arrive at an estimate of the probabilities. Although such an approach is feasible, it is likely that humans will often use shortcuts. For instance, Dougherty, Gettys, and Ogden (1999) provided an elegant proposal of how people can estimate probabilities in a relatively effortless manner.

Shanks (1995) argued that the effect of trial order on HCL is incompatible with the hypothesis that people calculate and compare probabilities when learning about cue-outcome relations (see De Houwer, Vandorpe, \& Beckers, 2005, for a discussion of some other possible criticisms). Many studies have shown that contingency judgments are often influenced more by recent trials than 
by trials that were presented at the beginning of the learning phase (e.g., Lopez, Shanks, Almaraz, \& Fernandez, 1998). Such recency effects should not occur when contingency judgments are based on probabilities because conditional probabilities are not affected by trial order. But more recent studies suggest that trial-order effects depend on beliefs that participants have about what information is relevant in a given test context (Matute, Vegas, \& De Marez, 2002). For instance, if participants are required to give a contingency judgment after every trial, they are inclined to think that their judgments should reflect the most recent information that they received. If, however, they are asked to give only one rating at the end of all trials, this encourages them to think that all the presented information should be taken into account when making the judgment. In line with this prediction, recency effects are much stronger when judgments need to be made on each trial than when only one judgment at the end of the learning phase is required (e.g., Collins \& Shanks, 2002; see Matute et al., 2002, for an in-depth discussion of this issue). Research on trial-order effects is thus compatible with the idea that people arrive at conscious propositional knowledge about cue-outcome relations by calculating and comparing probabilities on the basis of observed events.

Human autonomic conditioning. Studies on human autonomic conditioning are procedurally identical to HCL studies except that learning is indexed by means of nonverbal autonomic responses (e.g., galvanic skin responses; GSR) instead of explicit ratings. Mitchell and Lovibond (2002) and Lovibond (2003) demonstrated that higher order reasoning also plays an important role in human autonomic conditioning.

Mitchell and Lovibond (2002) used colored squares as conditioned stimuli (CSs), electric shocks as USs, and measured associatively induced changes in GSR to the CSs. One color (Color A) was followed by a shock both when it was presented on its own $\left(\mathrm{A}^{+}\right)$and when it was presented in compound with a second colored square $\left(\right.$ Color $\left.\mathrm{T} ; \mathrm{AT}^{+}\right)$. During a training phase that preceded the learning phase, participants saw two other colors that were also always followed by the shock when presented on their own $\left(\mathrm{X}^{+}, \mathrm{Y}^{+}\right)$and when presented in compound $\left(\mathrm{XY}^{+}\right)$. However, for half of the participants, the shock was twice as intense on the $\mathrm{XY}^{+}$trials as on the $\mathrm{X}^{+}$and $\mathrm{Y}^{+}$trials, whereas for the other participants, the intensity of the shock was the same on the $\mathrm{XY}^{+}$trials as on the $\mathrm{X}^{+}$ and $\mathrm{Y}^{+}$trials. Increasing the intensity of the shock on the XY trials should encourage participants to believe that the effects of the various CSs are additive. Mitchell and Lovibond found blocking only when the intensity of the shock on $\mathrm{XY}^{+}$trials was higher than on $\mathrm{X}^{+}$and $\mathrm{Y}^{+}$ trials. This clearly supports the thesis that higher order reasoning plays an important role in human autonomic conditioning.

In another study on human autonomic conditioning, Lovibond (2003) found that release from overshadowing occurred even (1) when $\mathrm{AT}^{+}$and $\mathrm{A}^{-}$were described verbally rather than actually presented and (2) when the
$\mathrm{AT}^{+}$trials were actually presented but the subsequent $\mathrm{A}^{-}$ trials were replaced by the verbal message that A was a safe cue that would not be followed by the US. This is in line with the idea that participants can form conscious propositional knowledge on the basis of either observation or instruction and combine this knowledge to make inferences about CS-US relations.

Animal conditioning. One might be inclined to believe that higher order reasoning processes cannot play a role in animal conditioning simply because nonhuman animals are incapable of such reasoning. But as Brewer (1974, p. 29) pointed out, it might well be interesting to entertain the hypothesis that at least some nonhuman animals also use controlled reasoning processes when learning about associations. To test this hypothesis, one could examine whether findings that provide strong support for the role of higher order reasoning processes in human learning (e.g., the impact of secondary tasks and ceiling effects on blocking) can be replicated in studies with other animals. In other words, research on the role of higher order reasoning in HCL could provide an interesting source of inspiration for research on associative learning in nonhuman animals.

\section{On the Processes That Underlie Higher Order Reasoning}

We have described higher order reasoning as reflective, rule based, deliberative, and controlled but did not specify where the ability to reason comes from or how this process is actually executed. Some might argue that the ability to reason could well originate from the operation of automatic associative processes similar to the ones that form the core of traditional models of associative learning (e.g., Rescorla \& Wagner, 1972). Although it might be possible to construct an associative learning model of reasoning, we do not know of any existing associative model that even comes close to being a model of reasoning or to being a starting point for such a model. Even if such a model could be constructed, it should be able to simulate the fact that reasoning has the functional properties of a controlled process (e.g., effortful, intentional, conscious, controllable). Such a model would therefore be functionally equivalent to a higher order reasoning account.

In order to understand the origins and nature of higher order reasoning, it would be best to turn to the extensive literature on reasoning in cognitive psychology. It would take us too far to even sketch the insights that can be found in this literature. We do want to emphasize, however, that a closer interaction is needed between research on associative learning and research on reasoning. We are convinced that such an interaction will help to increase our understanding of associative learning.

\section{Limitations of Higher Order Reasoning Accounts}

It should be clear that existing higher order reasoning accounts such as those of Waldmann (e.g., Waldmann \& Walker, 2005) and Lovibond (e.g., Lovibond, 2003) are 
limited in several ways. For instance, they are largely silent about how higher order reasoning is actually achieved or how people learn the causal models or deductive rules on which reasoning relies. A related problem is that the accounts are formulated in a largely verbal manner rather than being formalized mathematically. As a result, they lack the precision that is offered by many formalized associative models of learning (e.g., Rescorla \& Wagner, 1972). The most troubling implication of this lack of precision is that it becomes difficult to refute higher order reasoning accounts. There are so many free parameters that it is often possible to come up with a post hoc explanation of findings that are at first sight problematic for a reasoning account. Although an inspection of the recent literature on HCL shows that researchers also do not hesitate to make post hoc adjustments to formalized associative models (e.g., Melchers et al., 2004; Van Hamme \& Wasserman, 1994), it is true that the lack of precision of the existing higher order reasoning accounts makes them particularly flexible, perhaps even too flexible.

Despite these limitations, higher order reasoning accounts do have the important merit that they allow one to make new testable predictions. The core assumption of these models is that learning depends on higher order reasoning. Because higher order reasoning is a controlled process that operates on conscious propositional knowledge, one can predict that learning that depends on reasoning will have the functional properties of a controlled process (i.e., effortful, conscious, time-consuming, intentional, controllable) and will depend on the conscious propositional knowledge that people hold at a particular moment in time. As we have seen above, many of these predictions have been verified and several still await testing. Although higher order reasoning accounts thus clearly have scientific merit, one could argue that they are currently too poorly specified to be regarded as true scientific theories. An important challenge for the future is to add more detail to and formalize the current reasoning accounts. Such developments could again lead to new insights in HCL.

\section{CONCLUSION}

Research on cue competition has led to many developments in theories of HCL and associative learning in general. We have reviewed evidence that strongly suggests that higher order reasoning is a main source of cue competition effects in HCL. We have also described how higher order reasoning can account for other phenomena in HCL and human autonomic conditioning. Higher order reasoning thus appears to be a major component of associative learning in general.

\section{REFERENCES}

Aitken, M. R. F., Larkin, M. J. W., \& Dickinson, A. (2001). Reexamination of the role of within-compound associations in the ret- rospective revaluation of causal judgements. Quarterly Journal of Experimental Psychology, 54B, 27-51.

BREWER, W. F. (1974). There is no convincing evidence of conditioning in adult humans. In W. B. Weimer \& D. S. Palermo (Eds.), Cognition and the symbolic processes (pp. 1-42). Hillsdale, NJ: Erlbaum.

Cheng, P. W. (1997). From covariation to causation: A causal power theory. Psychological Review, 104, 367-405.

Cheng, P. W., \& Holyoak, K. J. (1995). Complex adaptive systems as intuitive statisticians: Causality, contingency, and prediction. In J.-A. Meyer \& H. Roitblat (Eds.), Comparative approaches to cognition (pp. 271-302). Cambridge, MA: MIT Press.

Cheng, P. W., \& Novick, L. R. (1990). A probabilistic contrast model of causal induction. Journal of Personality \& Social Psychology, 58, 545-567.

Cobos, P. L., López, F. J., Caño, A., Almaraz, J., \& Shanks, D. R. (2002). Mechanisms of predictive and diagnostic causal induction. Journal of Experimental Psychology: Animal Behavior Processes, 28,331-346.

Collins, D. J., \& Shanks, D. R. (2002). Momentary and integrative response strategies in causal judgment. Memory \& Cognition, 30, $1138-1147$.

DE HOUWER, J. (2002). Forward blocking depends on retrospective inferences about the presence of the blocked cue during the elemental phase. Memory \& Cognition, 30, 24-33.

De Houwer, J., \& BECKERS, T. (2002a). Higher-order retrospective revaluation in human causal learning. Quarterly Journal of Experimental Psychology, 55B, 137-151.

De Houwer, J., \& BeCKers, T. (2002b). A review of recent developments in research and theory on human contingency learning. Quarterly Journal of Experimental Psychology, 55B, 289-310.

DE HOuwER, J., \& BECKERS, T. (2002c). Second-order backward blocking and release from overshadowing in human causal learning. Experimental Psychology, 49, 27-33.

De Houwer, J., \& BECKERS, T. (2003). Secondary task difficulty modulates forward blocking in human contingency learning. Quarterly Journal of Experimental Psychology, 56B, 345-357.

De Houwer, J., BecKers, T., \& Glautier, S. (2002). Outcome and cue properties modulate blocking. Quarterly Journal of Experimental Psychology, 55A, 965-985.

De Houwer, J., Vandorpe, S., \& Beckers, T. (2005). On the role of controlled cognitive processes in human associative learning. In A. Wills (Ed.), New directions in human associative learning (pp. 4163). Mahwah, NJ: Erlbaum.

Denniston, J. C., Savastano, H. I., \& Miller, R. R. (2001). The extended comparator hypothesis: Learning by contiguity, responding by relative strength. In R. R. Mowrer \& S. B. Klein (Eds.), Handbook of contemporary learning theories (pp. 65-117). Mahwah, NJ: Erlbaum.

Dickinson, A. (2001). The 28th Bartlett Memorial Lecture: Causal learning. An associative analysis. Quarterly Journal of Experimental Psychology, 54B, 3-25.

DICKINSON, A., \& BURKE, J. (1996). Within-compound associations mediate the retrospective revaluation of causality judgements. Quarterly Journal of Experimental Psychology, 49B, 60-80.

DiCKInSON, A., ShanKs, D., \& Evenden, J. (1984). Judgement of actoutcome contingency: The role of selective attribution. Quarterly Journal of Experimental Psychology, 36A, 29-50.

Dougherty, M. R., Gettys, C. F., \& Ogden, E. E. (1999). MINERVA_DM: A memory processes model for judgments of likelihood. Psychological Review, 106, 180-209.

Evans, J. ST. B. T., \& OvER, D. E. (1996). Rationality and reasoning. Hove, U.K.: Psychology Press.

Glautier, S. (2002). Spatial separation of target and competitor cues enhances blocking of human causality judgements. Quarterly Journal of Experimental Psychology, 55B, 121-136.

KRUSCHKE, J. K. (2001). Toward a unified model of attention in associative learning. Journal of Mathematical Psychology, 45, 812-863.

KruschKe, J. K., \& BLAIR, N. J. (2000). Blocking and backward blocking involve learned inattention. Psychological Bulletin \& Review, 7, 636-645. 
Larkin, M. J. W., Aitken, M. R. F., \& Dickinson, A. (1998). Retrospective revaluation of causal judgments under positive and negative contingencies. Journal of Experimental Psychology: Learning, Memory, \& Cognition, 24, 1331-1352.

Le Pelley, M. E., \& Mclaren, I. P. L. (2003). Learned associability and associative change in human causal learning. Quarterly Journal of Experimental Psychology, 56B, 68-79.

Lopez, F. J., Shanks, D. R., AlmaraZ, J., \& Fernandez, P. (1998). Effects of trial order on contingency judgments: A comparison of associative and probabilistic contrast accounts. Journal of Experimental Psychology: Learning, Memory, \& Cognition, 24, 672-694.

LoviBOND, P. F. (2003). Causal beliefs and conditioned responses: Retrospective revaluation induced by experience and by instruction. Journal of Experimental Psychology: Learning, Memory, \& Cognition, 29, 97-106.

LovibOND, P. F., BeEn, S.-L., Mitchell, C. J., Bouton, M. E., \& FroHART, R. (2003). Forward and backward blocking of causal judgment is enhanced by additivity of effect magnitude. Memory \& Cognition, 31, 133-142.

MACHO, S., \& BURKART, J. (2002). Recursive retrospective revaluation of causal judgments. Journal of Experimental Psychology: Learning, Memory, \& Cognition, 28, 1171-1186.

MACKINTOSH, N. J. (1975). A theory of attention: Variation in the associability of stimuli with reinforcement. Psychological Review, 82, 276-298.

Matute, H., Vegas, S., \& De MareZ, P.-J. (2002). Flexible use of recent information in causal and predictive judgment. Journal of Experimental Psychology: Learning, Memory, \& Cognition, 28, 714-725.

MELCHERS, K. G., LaChNIT, H., \& ShanKS, D. (2004). Within-compound associations in retrospective revaluation and in direct learning: A challenge for comparator theory. Quarterly Journal of Experimental Psychology, 57B, 25-54.

MiLleR, R. R., \& MATZEL, L. D. (1988). The comparator hypothesis: A response rule for the expression of associations. In G. H. Bower (Ed.), The psychology of learning and motivation (Vol. 22, pp. 5192). San Diego: Academic Press.

Mitchell, C. J., \& Lovibond, P. F. (2002). Backward and forward blocking in human electrodermal conditioning: Blocking requires an assumption of outcome additivity. Quarterly Journal of Experimental Psychology, 55B, 311-330.

PEARCE, J. M., \& Bouton, M. E. (2001). Theories of associative learning. Annual Review of Psychology, 52, 111-139.

REsCoRLA, R. A., \& WAGNER, A. R. (1972). A theory of Pavlovian conditioning: Variations in the effectiveness of reinforcement and nonreinforcement. In A. H. Black \& W. F. Prokasy (Eds.), Classical conditioning II: Current research and theory (pp. 64-99). New York: Appleton.

SHANKS, D. R. (1985). Forward and backward blocking in human contingency judgement. Quarterly Journal of Experimental Psychology, 37B, 1-21.

SHANKs, D. R. (1995). Is human learning rational? Quarterly Journal of Experimental Psychology, 48A, 257-279.

SHANKS, D. R., \& LóPEZ, F. J. (1996). Causal order does not affect cue selection in human associative learning. Memory \& Cognition, 24, 511-522.

SLOMAN, S. A. (1996). The empirical case for two systems of reasoning. Psychological Bulletin, 119, 322.

Smith, E. R., \& DeCoster, J. (2000). Dual process models in social and cognitive psychology: Conceptual integration and links to underlying memory systems. Personality \& Social Psychology Review, 4, 108-131.

StRaCK, F., \& Deutsch, R. (2004). Reflective and impulsive determinants of social behavior. Personality \& Social Psychology Review, 8 , 220-247.

TANGEN, J. M., \& Allan, L. G. (2004). Cue-interaction and judgments of causality. Memory \& Cognition, 32, 107-124.

VAn Hamme, L. J., \& Wasserman, E. A. (1994). Cue competition in causality judgements: The role of nonpresentation of compound stimulus elements. Learning \& Motivation, 25, 127-151.

WaLdMANN, M. R. (2000). Competition among causes but not effects in predictive and diagnostic learning. Journal of Experimental Psychology: Learning, Memory, \& Cognition, 26, 53-76.

WALDMANN, M. R., \& HolYOAK, K. J. (1992). Predictive and diagnostic learning within causal models: Asymmetries in cue competition. Journal of Experimental Psychology: General, 121, 222-236.

WALDMANN, M. R., \& WALKER, J. M. (2005). Competence and performance in causal learning. Learning \& Behavior, 33, 211-229.

Wasserman, E. A., \& Berglan, L. R. (1998). Backward blocking and recovery from overshadowing in human causal judgement: The role of within-compound associations. Quarterly Journal of Experimental Psychology, 51B, 121-138.

Williams, D. A., SAGNESS, K. E., \& McPhee, J. E. (1994). Configural and elemental strategies in predictive learning. Journal of Experimental Psychology: Learning, Memory, \& Cognition, 20, 694-709.

WU, M., \& CHENG, P. W. (1999). Why causation need not follow from statistical covariation: Boundary conditions for the evaluation of generative and preventative causal powers. Psychological Science, 10, 93-97.

\section{NOTES}

1. Waldmann (2000) referred to this effect as partial blocking. However, it could be due either to overshadowing ( $\mathrm{T}$ is presented in compound with another cue) or to blocking ( $\mathrm{T}$ is presented in compound with another cue that is itself followed by the outcome). But regardless of the true nature of the effect, it is clear that instructions about the causal nature of the cues have an impact on the effect. Also, De Houwer et al. (2002) demonstrated that true blocking (i.e., lower ratings for T after $\mathrm{AT}^{+}$and $\mathrm{A}^{+}$trials than after only $\mathrm{AT}^{+}$trials) was also influenced by the causal nature of the cues (i.e., causes or predictors of the outcome).

2. Note that when we talk about associative learning, we do not mean the formation of associative links between representations. Rather, we use the term associative learning to refer to changes in behavior that are due to the paired presentation of stimuli. That is, we use the term to refer to an observable effect rather than to theoretical ideas about how this effect is produced (e.g., the formation of associations in memory). 